\title{
Forward production of heavy flavour hadrons in p-p collisions and intrinsic charm and bottom in proton
}

\author{
D. A. Artemenkov, V. A. Bednyakov, G. I. Lykasov ${ }^{1}$ \\ JINR, Dubna, Moscow region, 141980, Russia, \\ ${ }^{1}$ E-mail: lykasov@jinr.ru
}

DOI: http://dx.doi.org/10.5689/UA-PROC-2010-09/46

\begin{abstract}
We present the theoretical results on the forward $\Lambda_{b}$ production in $p p$ collisions obtained within the soft QCD, namely the quark gluon string model, at LHC energies. It can give us useful information on the Regge trajectories of the bottom mesons. An opportunity to find the information on the intrinsic charm and bottom in the proton from the analysis of the forward heavy flavour hadron production in $p p$ collisions is discussed.
\end{abstract}

\section{Introduction}

As is well known, there are successful phenomenological approaches for describing the soft hadron-nucleon, hadron-nucleus and nucleus-nucleus interactions at high energies based on the Regge theory and the $1 / N$ expansion in QCD, for example the quark-gluon string model (QGSM) [1]. and the dual parton model (DPM) [2]. The main components of the QGSM and the DPM are the quark distributions in a hadron and the the fragmentation functions (FF) describing quark fragmentation into hadrons. These are expressed in terms of intercepts of the Regge trajectories $\alpha_{R}(0)$. The largest uncertainty in the calculations of the cross sections for the yields of heavy flavours in these models is mainly due to the absence of any reliable information on the transfer $t$ dependence of the Regge trajectories of heavy quarkonia $(Q \bar{Q})$. Assuming linearity of the $(Q \bar{Q})$ trajectories, the intercepts turn out to be low, $\alpha_{\psi}(0)=-2.2$, $\alpha_{\Upsilon}(0)=-8,-16$, and so the contribution of the peripheral mechanism decreases very rapidly with increasing quark mass, Accordingly, the finding of the $t$-dependence for $\alpha_{(Q \bar{Q})}(t)$ in the region $0 \leq t \leq M_{(Q \bar{Q})}^{2}$ and estimations of their intercepts become especially important. In this paper we present the results on the beauty baryon production, in particular $\Lambda_{b}$, in $p p$ collisions at LHC energies and small $p_{t}$ within the QGSM to find the information on the Regge trajectories of the bottom $(b \bar{b})$ mesons and the fragmentation functions $(\mathrm{FF})$ of all the quarks and diquarks to this baryon. 

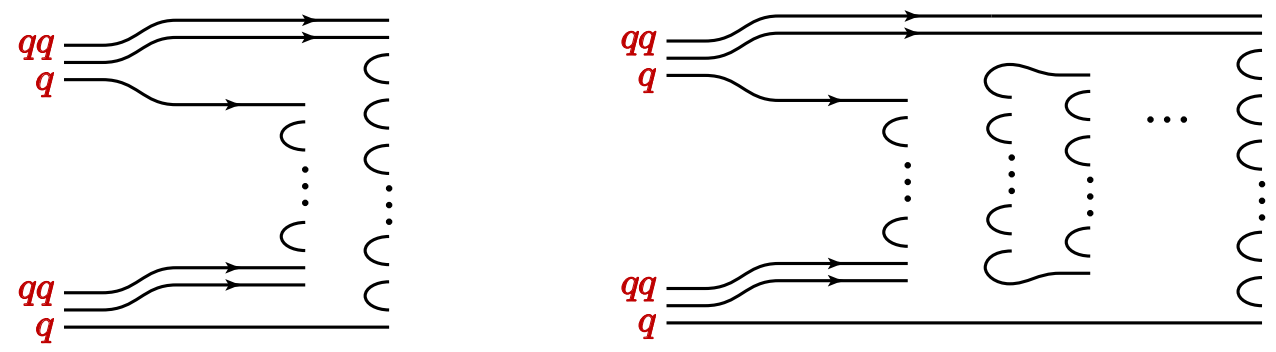

Figure 1: The one-cylinder graph (left) and the multicylinder graph (right) for the inclusive $p p \rightarrow h X$ process.

\section{General formalism}

The general form for the invariant inclusive hadron spectrum within the QGSM is $[1,2]$

$$
E \frac{d \sigma}{d^{3} \mathbf{p}} \equiv \frac{2 E^{*}}{\pi \sqrt{s}} \frac{d \sigma}{d x d p_{t}^{2}}=\sum_{n=1}^{\infty} \sigma_{n}(s) \phi_{n}\left(x, p_{t}\right),
$$

where $E, \mathbf{p}$ are the energy and the three-momentum of the produced hadron $h$ in the laboratory system (l.s.); $E^{*}, s$ are the energy of $h$ and the square of the initial energy in the c.m.s of $p p$; $x, p_{t}$ are the Feynman variable and the transverse momentum of $h ; \sigma_{n}$ is the cross section for production of the $n$-Pomeron chain (or $2 n$ quark-antiquark strings) decaying into hadrons, calculated within the quasi- "eikonal approximation" [3]; $n=1$ corresponds to the left graph of Fig.1, whereas $n>1$ corresponds to the right graph of Fig.1. Actually, the function $\phi_{n}\left(x, p_{t}\right)$ is the convolution of the quark (diquark) distributions and the FF, see the details in [1] and $[2,4]$.

$$
\begin{array}{r}
\phi_{n}^{p p}(x)=F_{q q}^{(n)}\left(x_{+}\right) F_{q_{v}}^{(n)}\left(x_{-}\right)+F_{q_{v}}^{(n)}\left(x_{+}\right) F_{q q}^{(n)}\left(x_{-}\right)+ \\
2(n-1) F_{q_{s}}^{(n)}\left(x_{+}\right) F_{\bar{q}_{s}}^{(n)}\left(x_{-}\right),
\end{array}
$$

where $x_{ \pm}=\frac{1}{2}\left(\sqrt{x^{2}+x_{t}^{2}} \pm x\right)$, and

$$
F_{\tau}^{(n)}\left(x_{ \pm}\right)=\int_{x_{ \pm}}^{1} d x_{1} f_{\tau}^{(n)}\left(x_{1}\right) G_{\tau \rightarrow h}\left(\frac{x_{ \pm}}{x_{1}}\right)
$$

Here $\tau$ means the flavour of the valence (or sea) quark or diquark, $f_{\tau}^{(n)}\left(x_{1}\right)$ is the quark distribution function depending on the longitudinal momentum fraction $x_{1}$ in the $n$-Pomeron chain; $G_{\tau \rightarrow h}(z)=z D_{\tau \rightarrow h}(z), D_{\tau \rightarrow h}(z)$ is the FF of a quark (antiquark) or diquark of flavour $\tau$ into a hadron $h$ (charmed or bottom hadron in our case). All the details of the calculation of Eq.(1) and the interaction function $\phi_{n}\left(x, p_{t}\right)$ can be found in $[6,7]$.

\section{Results and discussion}

Fig.2 illustrates the sensitivity of the inclusive spectrum $d \sigma / d x$ of the produced charmed baryons $\Lambda_{c}$ to the different values for the intercept $\alpha_{\psi}(0)$ of the $\psi(c \bar{c})$ Regge trajectory. The solid line 
corresponds to $\alpha_{\psi}(0)=0$, and the dashed curve corresponds to $\alpha_{\psi}(0)=-2.18$. One can see that there are difference between the R608 [10] and R422[11] experimental data. As is shown in [7], the R608 data are more adequate to our calculations at $\alpha_{\Psi}(0)=0$, see the solid line in Fig.2. Experimentally one can measure the differential cross section $d \sigma / d \xi_{p} d t_{p} d M_{J / \Psi}$, where $\xi_{p}=\Delta p / p$ is the energy loss, $t_{p}=\left(p_{i n}-p_{1}\right)^{2}$ is the four-momentum transfer, $M_{J / \Psi}$ is the effective mass of the $J / \Psi$-meson. The detailed predictions on these reactions are presented in

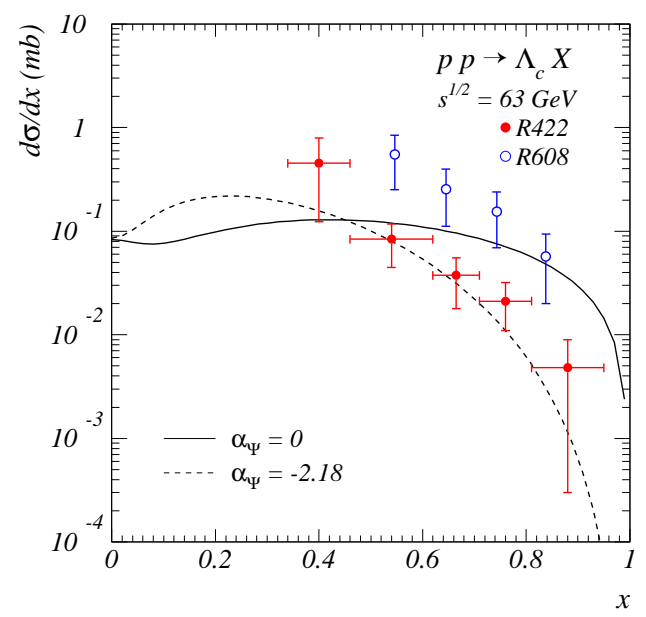

Figure 2: The differential cross section $d \sigma / d x$ for the inclusive process $p p \rightarrow \Lambda_{c} X$ at $\sqrt{s}=$ $62 \mathrm{GeV}$. The solid line corresponds to $\alpha_{\psi}(0)=0$, and the dashed curve corresponds to $\alpha_{\psi}(0)=$ -2.18 . The open circles correspond to the R608 experiment [10], and the dark circles correspond to the R422 experiment [11].

[7], where it is shown that all the observables are very sensitive to the value of intercept $\alpha_{\Upsilon}(0)$ of the $\Upsilon(b \bar{b})$ Regge trajectory. The upper limit of our results is reached at $\alpha_{\Upsilon}(0)=0$, when this Regge trajectory as a function of the transfer $t$ is nonlinear. Using the hadron detector at the CMS and the TOTEM one could register the decay $\Lambda_{b}^{0} \rightarrow J / \Psi \Lambda^{0} \rightarrow \mu^{+} \mu^{-} \pi^{-} p$ by detecting two muons and one proton emitted forward. However, the acceptance of the muon detector is $10^{0} \leq \theta_{\mu} \leq 170^{0}$ [8], where, according to our calculations, the fraction of this events is to low. On the other hand, the electromagnetic calorimeter at the CMS is able to measure the dielectron pairs $e^{+} e^{-}$in the acceptance about $1^{0} \leq \theta_{e\left(e^{+}\right)} \leq 179^{0}$ [9]. In Fig.3 the twodimensional distribution over $E_{p}$ and $\theta_{p}$ for the reaction $p p \rightarrow \Lambda_{b} X \rightarrow J / \Psi \Lambda^{o} X \rightarrow e^{+} e^{-} p \pi^{-} X$ is presented. The rate of these events is about 0.74 percent $(2.22 \mathrm{nb})$. This could be reliable using the TOTEM together with the CMS [12].

The ATLAS is able also to detect $e^{+} e^{-}$by the electromagnetic calorimeter in the interval $1^{0} \leq \theta_{e\left(e^{+}\right)} \leq 179^{0}[8]$ and the neutrons emitted forward at the angles $\theta_{n} \leq 0.1 \mathrm{mrad}$ [13]. In Fig.4 we present the prediction for the reaction $p p \rightarrow \Lambda_{b} X \rightarrow J / \Psi \Lambda^{o} X \rightarrow e^{+} e^{-} n \pi^{0} X$, that could be reliable at the ATLAS experiment. The rate of these events is about 0.015 percent (45 pb).

The TOTEM [14] together with the CMS might be able to measure the channel $\Lambda_{b} \rightarrow$ $J / \Psi \Lambda^{0} \rightarrow e^{+} e^{-} \pi^{-} p$ (the integrated cross-section is about $0.2-0.3 \mu \mathrm{b}$ at $\alpha_{\Upsilon}(0)=0$ and 
smaller at $\left.\alpha_{\Upsilon}(0)=-8\right)$. The T2 and T1 tracking stations of the TOTEM apparatus have their angular acceptance in the intervals $3 \mathrm{mrad}<\theta<10 \mathrm{mrad}$ (corresponding to $6.5>\eta>5.3$ ) and $18 \mathrm{mrad}<\theta<90 \mathrm{mrad}$ (corresponding to $4.7>\eta>3.1$ ) respectively, and could thus detect $42 \%$ of the muons from the $J / \Psi$ decay. In the same angular intervals, $36 \%$ of the $\pi^{-}$and $35 \%$ of the protons from the $\Lambda^{0}$ decay are expected. According to a very preliminary estimate [12], protons with energies above $3.4 \mathrm{TeV}$ emitted at angles smaller than $0.6 \mathrm{mrad}$ could be detected in the Roman Pot station at $147 \mathrm{~m}$ from IP5 [14, 12]. In the latter case, the reconstruction of the proton kinematics may be possible, whereas the trackers T1 and T2 do not provide any momentum or energy information. Future detailed studies are to establish the full event topologies with all correlations between the observables in order to assess whether the signal events can be identified and separated from backgrounds. These investigations should also include the CMS calorimeters HF and CASTOR which cover the same angular ranges as T1 and T2 respectively [12].

\section{Intrinsic charm and bottom in proton}

Let us discuss an opportunity to find the information on the distributions of intrinsic charm and beauty in proton from the analysis of the forward production of heavy flavour baryons in $p p$ collisions at LHC. Note that all the sea quark distributions in the proton calculated within the QGSM give the contributions only to the multi-Pomeron graphs at $n \geq 2$, see Fig.1 (right), and do not contribute to the one-Pomeron graph (Fig.1(left)), according to Eq.(2). The one-Pomeron graph results in the main contribution at large values of $x$ because $\sigma_{n}$ decreases very fast when $n$ increases [3]. Therefore, as our calculations showed recently [5]-[7], the sea charm and beauty quark distributions, in fact, result in very small contributions to the inclusive spectra of the charmed and beauty hadrons at different values of $x$ and at not large $p_{t}$. For the forward heavy flavour hadron production this contribution is smaller, therefore, we neglect this. The sea charm and beauty quark distributions are not the same as the distributions of the intrinsic charm and bottom quarks in the proton, which have the forms like the valence quark distributions $[15,16]$. If we want to include the intrinsic charm and bottom quark distributions within the QGSM scheme we should include them calculating the one-Pomeron graph (Fig.1(left)), e.g., at $n=1$. It can increase the rates of events for both reactions $p p \rightarrow \Lambda_{b} X \rightarrow e^{+} e^{-} p \pi^{-} X$ and $p p \rightarrow \Lambda_{b} X \rightarrow e^{+} e^{-} n \pi^{0} X$ when the final proton or neutron are emitted forward because the one-Pomeron graph (Fig.1(left) results a main contribution to the $\Lambda_{b}$ spectrum in this kinematics. However, as shown recently [17], the intrinsic charm in the proton can result in a sizable contribution to the forward charmed meson production. As is shown in [16], the probability to find the intrinsic charm in the proton is not more than 0.5 percent. However, the probability of the intrinsic bottom in the proton is suppressed by a factor $m_{c}^{2} / m_{b}^{2} \simeq 0.1$ [18], where $m_{c}$ and $m_{b}$ are the masses of the charmed and bottom quarks. Therefore, the contribution of the intrinsic bottom in the proton to the discussed reaction can be suppressed in comparison to the intrinsic charm contribution about ten times. Anyway, it would be interesting to study this problem more carefully.

\section{Conclusion}

We analyzed the production of charmed and beauty baryons in proton-proton collisions at high energies within soft QCD, namely the quark-gluon string model (QGSM). This approach can 
describe rather satisfactorily the charmed baryon production in $p p$ collisions $[6,7]$. It allows us to apply the QGSM to studying the beauty baryon production in $p p$ collisions. We focus mainly on the analysis of the forward $\Lambda_{b}$ production in $p p$ collisions at LHC energies and got some predictions which could be reliable at the TOTEM and ATLAS experiments at CERN. We present the predictions for the reaction $p p \rightarrow \Lambda_{b} X \rightarrow e^{+} e^{-} p \pi^{-} X$ that could be reliable at the TOTEM together with the CMS, and for the process $p p \rightarrow \Lambda_{b} X \rightarrow e^{+} e^{-} n \pi^{0} X$ which can be reliable at the ATLAS experiment using the ZDC. Our predictions using the nonlinear Regge trajectories of $c \bar{c}$ and $b \bar{b}$ mesons can be considered as the upper limit of the QGSM calculations without the intrinsic charm and bottom in the proton. [14, 12].

\section{Acknowledgments}

We are very grateful to V.V.Lyubushkin for a help in the MC calculations. We also thank M. Deile, P. Grafström, and N.I. Zimin for extremely useful help related to the possible experimental check of the suggested predictions at the LHC and the preparation of this paper. We are also grateful to D.Denegri, K. Eggert, S.Lami, T.Lomtadze, F.Palla, O.V.Teryaev, M. Poghosyan and S.White for very useful discussions. This work was supported in part by the Russian Foundation for Basic Research grant N: 08-02-01003.

\section{References}

[1] A. B. Kaidalov and K. A. Ter-Martirosyan, Phys. Lett. B 116 (1982) 489, [arXiv:hep-ph/0909.5061].

[2] A. Capella, U. Sukhatme, C. I. Tan and J. Tran Than Van Phys. Rep. 236 (1994) 225, [arXiv:hep$\mathrm{ph} / 0909.5061]$.

[3] K. A. Ter-Martirosyan, Phys. Lett. B44 (1973) 377.

[4] A. B. Kaidalov O. I. Piskunova, Z.Phys. C30(1986)145.

[5] G. I. Lykasov, Z. M. Karpova, M. N. Sergeenko and V. A. Bednyakov, Europhys.Lett., 86 (2009) 61001; arXiv:hep-ph/0.812.3220.

[6] G. I. Lykasov, V. V. Lyubushkin and V. A. Bednyakov, Nucl. Phys. [Proc. Suppl.] B198 (2010) 165 [arXiv:hep-ph/0909.5061].

[7] V. A. Bednyakov, G. I. Lykasov and V. V. Lyubushkin, Europhys.Lett., 92 (2010) 31001; arXiv:hep$\mathrm{ph} / 1005.0559$.

[8] ATLAS Collaboration, Technical Design Report, ATLAS-TDR-017,CERN-LHCC-2005-022.

[9] CMS Collaboration J. Phys. G: Nucl. Part. Phys. 34 (2007) 995.

[10] P. Chauvat, et al. Phys.Lett. B199 (1987) 304.

[11] G. Bari, et al. Nuovo Cim. A104(1991) 571.

[12] M. Deile, Private communication; H. Niewiadomski, TOTEM-NOTE, 002 (2009).

[13] ATLAS Collaboration, Letter of Intent "Zero Degree Calorimeters".

[14] TOTEM Collaboration, Technical Design Report, (2004),CERN-LHCC-2004-002; Addendum CERNLHCC-2004-020, "The Totem Experiment At The CERN Large Hadron Collider", JINST 32008 S08007.

[15] S. L. Brodsky, P. Hoyer and N. Sakai, Phys.Lett. B 593 (1980) 451.

[16] J. Pumplin, Phys.Rev. D73 (2006) 114015.

[17] V. P. Goncalves, F. S. Navarra and T. Ullrich, arXiv:hep-ph/0805.0810.

[18] M. V. Polyakov, A. Shafer, O. V. Teryaev, Phys.Rev. D60 (1999) 051502. 


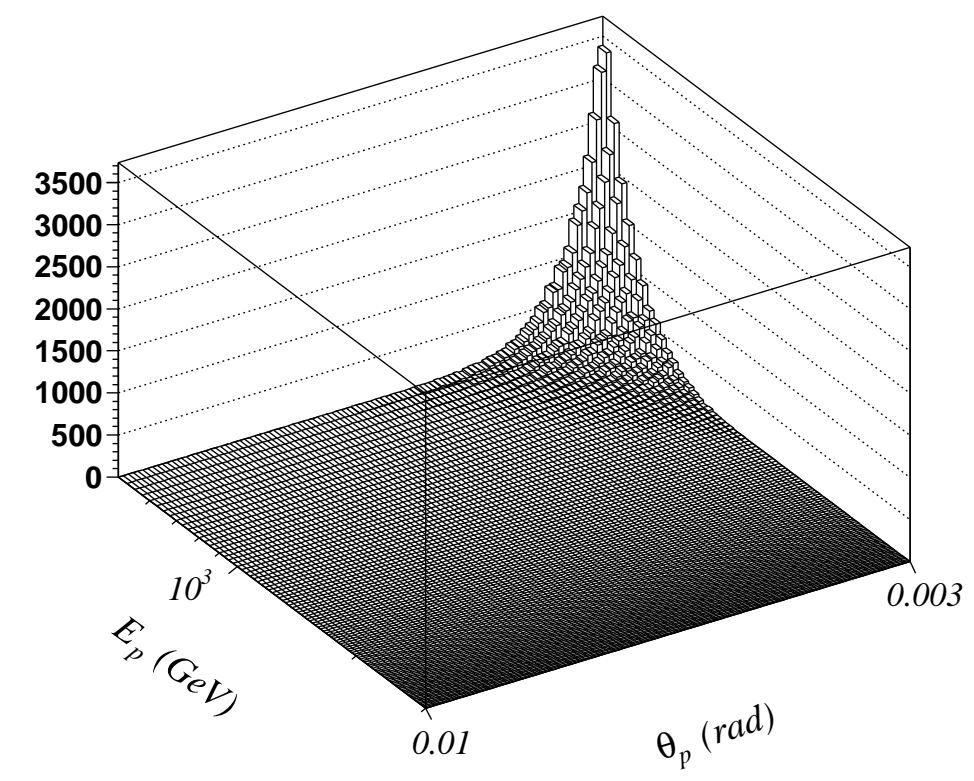

Figure 3: The two-dimensional distribution over $\theta_{p}$ and $E_{p}$ in the inclusive process $p p \rightarrow$ $\Lambda_{b} X \rightarrow J / \Psi \Lambda^{o} X \rightarrow e^{+} e^{-} p \pi^{-} X$ at $\sqrt{s}=10 \mathrm{TeV}$ at $\alpha_{\Upsilon}(0)=0$, when $500 G e V \leq E_{p} \leq 4 T e V$ and $3 \mathrm{mrad} \leq \theta_{p} \leq 10 \mathrm{mrad}$. The rate of these events is about 0.96 percent $(2.85 \mathrm{nb})$.

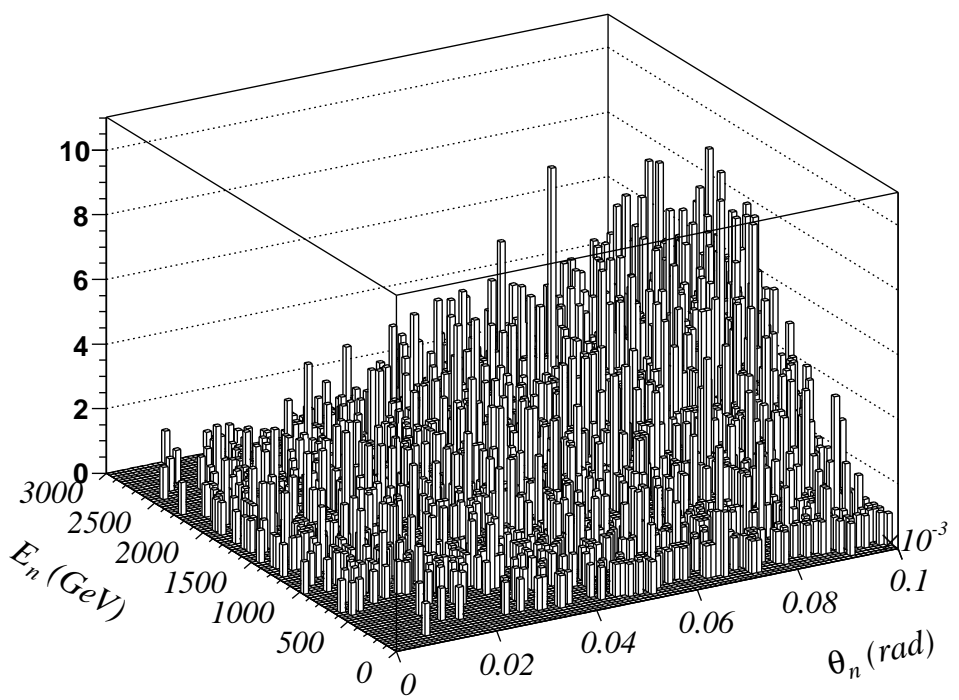

Figure 4: The two-dimensional distribution over $\theta_{p}$ and $E_{p}$ in the inclusive process $p p \rightarrow \Lambda_{b} X \rightarrow$ $J / \Psi \Lambda^{o} X \rightarrow e^{+} e^{-} n \pi^{0} X$ at $\sqrt{s}=10 \mathrm{TeV}$ at $\alpha_{\Upsilon}(0)=0$, when $\theta_{n} \leq 0.1$ mrad.. The rate of these events is about 0.015 percent $(45 \mathrm{pb})$. 